Stud. Univ. Babeş-Bolyai Math. 63(2018), No. 2, 269-284

DOI: $10.24193 /$ subbmath.2018.2.10

\title{
Some comments on a linear programming problem
}

\author{
Marcel Bogdan
}

\begin{abstract}
Besides the very known two exits of the Simplex Algorithm we consider two more cases when at least a solution exists and to decide whether or not the solution is unique. This situation occurred in a linear programming problem, on one hand applying the Simplex Algorithm and on the other hand using Matlab command linprog, that led to the case of unbounded solution set and its construction. Some necessary conditions on data are given so that the set of solutions to be boundedless.
\end{abstract}

Mathematics Subject Classification (2010): $90 \mathrm{C} 05$.

Keywords: Linear programming, simplex algorithm, multiple solutions.

\section{Introduction}

The standard form of a linear programming problem with data $c \in \mathbb{R}^{n}, A \in M_{m, n}(\mathbb{R})$, and $b \in \mathbb{R}^{m}$ is considered to be

$$
\left\{\begin{array}{l}
c^{T} x \longrightarrow \text { min } \\
A \cdot x=b \\
x \geq \mathbb{R}^{n} 0_{\mathbb{R}^{n}} .
\end{array}\right.
$$

There are some ways to obtain the solution(s) for this if any. Two algorithms are usually used: the simplex algorithm and the interior point algorithm. Theoretical aspects and examples are given in $[1,2,3,4,7]$, where simplex algorithm is exposed and $[6,8,9,10,11]$, where interior point algorithm can be found. On one side, there is the Simplex algorithm due to George Dantzig in 1947, that can be used to solve (1.1) with a pen and paper, on the other side there is a personal computer, a software (viewed as technology; Excel [12], Matlab [13], Octave [14, 15]), then pretending to understand the answer provided by the implemented algorithm. Comments about where mathematical programming extensions may be headed can be found in [5]. 
The commonly used simplex algorithm has two exits. Either provides a solution when the problem has one or establishes when there is no solution for the problem, the objective function being unbounded. When the algorithm is initiated it is not known if the admissible set is bounded. We propose a continuation of the algorithm when at least a solution exists and to decide whether or not the solution is unique. Our objective is to give a criteria for multiple solution (Proposition 3.2) and to indicate the steps when this is possible, in Algorithm 2.0, Step 4, case a3. This case has two branches:

a3 $\alpha$ ) for a convex hull of a finite set of solutions;

a3 $\beta$ ) for an unbounded set of solutions.

Some comments are related to the procedures and logistics to obtain a set of solutions, generated by an initial admissible point. In order to justify them and how these two different approaches work, the simplex algorithm along with Matlab optionally, we give the following example.

Example 1.1. Let us consider the linear programming problem

$$
\left\{\begin{array}{rrr}
x_{1}-x_{2}-3 x_{3}+5 x_{4} \rightarrow \min & \\
-x_{1}+x_{2}+x_{3}+x_{4} & =1 \\
x_{1}-2 x_{2}+3 x_{3} & +x_{5}=2 \\
x_{1}-x_{2} & +x_{6}=3 \\
x_{1}, \ldots, x_{6} \geq 0 &
\end{array}\right.
$$

with data $c^{T}=(1,-1,-3,5,0,0)$,

$$
A=\left(\begin{array}{rrrrrr}
-1 & 1 & 1 & 1 & 0 & 0 \\
1 & -2 & 3 & 0 & 1 & 0 \\
1 & -1 & 0 & 0 & 0 & 1
\end{array}\right), \quad \text { and } \quad b=\left(\begin{array}{l}
1 \\
2 \\
3
\end{array}\right)
$$

Denote by $A^{j} \in \mathbb{R}^{3}$ the $j$ th column of the matrix $A=\left(A^{j}\right)_{1 \leq j \leq 6}$. The canonical basis $\left\{A^{4}, A^{5}, A^{6}\right\}$ is primal admissible. We have $\mathcal{B}=\{4,5,6\}$ the set of indexes for which

$$
b=\sum_{j \in \mathcal{B}} \alpha_{0 j} A^{j} .
$$

By the simplex algorithm, after four steps (see Figure 1), one gets $\left\{A^{1}, A^{2}, A^{3}\right\}$ as optimal basis, so $\mathcal{B}=\{1,2,3\}$.

The solution $x^{B}$ has the components

$$
x_{j}^{B}= \begin{cases}\alpha_{0 j}, & j \in \mathcal{B} \\ 0, & \text { else }\end{cases}
$$

and for this case is

$$
\left(x^{B}\right)^{T}=(16,13,4,0,0,0) .
$$




\begin{tabular}{|c|c|c|c|c|}
\hline 1 & $A^{4}$ & $A^{5}$ & $A^{6}$ & \\
\hline$A^{1}$ & -1 & 1 & 1 & -6 \\
\hline$A^{2}$ & 1 & -2 & -1 & 6 \\
\hline$A^{3}$ & 1 & 3 & 0 & 8 \\
\hline & 1 & 2 & 3 & \\
\hline
\end{tabular}

\begin{tabular}{|c|c|c|c|c|}
\hline 2 & $A^{4}$ & $A^{3}$ & $A^{6}$ & \\
\hline$A^{1}$ & $-4 / 3$ & $1 / 3$ & 1 & $-26 / 3$ \\
\hline$A^{2}$ & $5 / 3$ & $-2 / 3$ & -1 & $34 / 3$ \\
\hline$A^{5}$ & $-1 / 3$ & $1 / 3$ & 0 & $-8 / 3$ \\
\hline & $1 / 3$ & $2 / 3$ & 3 & \\
\hline
\end{tabular}

\begin{tabular}{|c|c|c|c|c|}
\hline 3 & $A^{2}$ & $A^{3}$ & $A^{6}$ & \\
\hline$A^{1}$ & $-4 / 5$ & $-1 / 5$ & $1 / 5$ & $2 / 5$ \\
\hline$A^{4}$ & $3 / 5$ & $2 / 5$ & $3 / 5$ & $-34 / 5$ \\
\hline$A^{5}$ & $-1 / 5$ & $1 / 5$ & $-1 / 5$ & $-2 / 5$ \\
\hline & $1 / 5$ & $4 / 5$ & $16 / 5$ & \\
\hline
\end{tabular}

\begin{tabular}{|c|c|c|c|c|}
\hline 4 & $A^{2}$ & $A^{3}$ & $A^{1}$ & \\
\hline$A^{6}$ & & & 5 & -2 \\
\hline$A^{4}$ & & & 3 & -8 \\
\hline$A^{5}$ & -1 & 0 & -1 & 0 \\
\hline & 13 & 4 & 16 & \\
\hline
\end{tabular}

Figure 1. Simplex-primal-table(s)

In the fourth table we have $\alpha_{50}=0$, while $\alpha_{52}=-1, \alpha_{53}=0$, and $\alpha_{51}=-1$, therefore the multiple solution cannot be obtained by replacing one of the vectors from the optimal basis with $A^{5}$. The other six bases that are primal admissible $\left\{A^{1}, A^{2}, A^{3}\right\}$, $\left\{A^{1}, A^{2}, A^{4}\right\},\left\{A^{1}, A^{4}, A^{6}\right\},\left\{A^{2}, A^{3}, A^{6}\right\},\left\{A^{2}, A^{5}, A^{6}\right\},\left\{A^{3}, A^{4}, A^{6}\right\}^{1}$ and three bases that are dual admissible $\left\{A^{1}, A^{2}, A^{3}\right\},\left\{A^{1}, A^{3}, A^{5}\right\},\left\{A^{2}, A^{3}, A^{5}\right\}$, lead to the same optimal basis $\left\{A^{1}, A^{2}, A^{3}\right\}$, consequently to the same solution. Nevertheless, it is incorrectly to be considered as unique.

By Matlab we have

$$
\begin{aligned}
& >c=\left[\begin{array}{llllll}
1 & -1 & -3 & 5 & 0 & 0
\end{array}\right]
\end{aligned}
$$

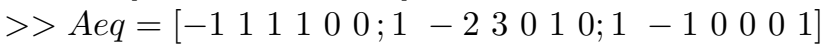

$$
\begin{aligned}
& >>\text { beq }=[1 ; 2 ; 3] \\
& >>l b=z \operatorname{eros}(1,6) \\
& >>\left[\begin{array}{ll}
x & z
\end{array}\right]=\operatorname{linprog}(c,[],[], \text { Aeq, beq, lb, [] }),
\end{aligned}
$$

with the solution generated by the interior point algorithm

$$
\left(x^{1}\right)^{T}=(102.8876,99.8876,4.0000,0,86.8876,0)
$$

with the minimal value $z=-9$.

It seems that solution $x^{1}$ cannot be obtained by the simplex algorithm. Consequently, in order to find the set of solutions, the simplex algorithm could and should be improved. Instead, the solution for (1.2) can be obtained analytically. From the first constraint $x_{4}=1+x_{1}-x_{2}-x_{3} \geq 0$ we have the equivalent linear programming

\footnotetext{
${ }^{1}$ none of the other five extremal points is optimum
} 
problem

$$
\left\{\begin{aligned}
3 x_{1}-3 x_{2}-4 x_{3} & \longrightarrow \min \\
-x_{1}+x_{2}+x_{3} & \leq 1 \\
x_{1}-2 x_{2}+3 x_{3} & \leq 2 \\
x_{1}-x_{2} & \leq 3 \\
x_{1}, x_{2}, x_{3} \geq 0 . &
\end{aligned}\right.
$$

Once again from the first constraint and the third one $x_{3} \leq 1+x_{1}-x_{2} \leq 4$. The function to be optimized is

$$
3 \cdot\left(x_{1}-x_{2}-x_{3}\right)-x_{3} \geq 3 \cdot(-1)-x_{3} \geq-3-4,
$$

its minimum value being attained when $x_{1}-x_{2}-x_{3}=-1$ and $x_{3}=4$, so

$$
x_{1}-x_{2}=3,
$$

at the intersection of the two planes. From

$$
\left\{\begin{aligned}
-x_{1}+x_{2}+x_{3} & =1 \\
x_{1}-x_{2} & =3
\end{aligned}\right.
$$

and from the second constraint of (1.3) we get $x_{2} \geq 13$. The complete solution for (1.3) is therefore $\left(x_{1}, x_{2}, x_{3}\right)=(\alpha+3, \alpha, 4)$, with $\alpha \geq 13$, or equivalently

$$
\left(x_{1}, x_{2}, x_{3}\right)=(16+\alpha, 13+\alpha, 4), \quad \text { with } \alpha \geq 0,
$$

thus $\left(x_{1}, x_{2}, x_{3}, x_{4}, x_{5}, x_{6}\right)=(16+\alpha, 13+\alpha, 4,0, \alpha, 0)$, with $\alpha \geq 0$, for (1.2).

Remark that, for $\varepsilon_{1}, \varepsilon_{2}>0,\left(16-\varepsilon_{1}, 13-\varepsilon_{2}, 4\right),\left(16-\varepsilon_{1}, 13,4\right),\left(16,13-\varepsilon_{2}, 4\right)$ are not solutions.

By Matlab we have

$$
\begin{aligned}
& >>c^{\prime}=\left[\begin{array}{lll}
3 & -3 & -4
\end{array}\right]
\end{aligned}
$$

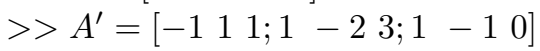

$$
\begin{aligned}
& >>b=[1 ; 2 ; 3] \\
& >l b=z \operatorname{eros}(1,3) \\
& >>\left[\begin{array}{ll}
x & z^{\prime}
\end{array}\right]=\operatorname{linprog}\left(c^{\prime}, A^{\prime}, b,[],[], l b,[]\right),
\end{aligned}
$$

with the solution generated by the interior point algorithm

$$
\left(x^{2}\right)^{T}=(116.3595,113.3595,4.0000),
$$

with the minimal value $z^{\prime}=-7$ (with $z=2 z^{\prime}+5$ ).

Let us denote by $f: \mathbb{R}^{6} \rightarrow \mathbb{R}$ and $f_{2}: \mathbb{R}^{3} \rightarrow \mathbb{R}$ the objective functions and by $S=\left\{x \in \mathbb{R}^{6} \mid A \cdot x=b, x \geq_{\mathbb{R}^{6}} 0_{\mathbb{R}^{6}}\right\}, S^{\prime}=\left\{x \in \mathbb{R}^{3} \mid A^{\prime} \cdot x \leq_{\mathbb{R}^{3}} b, x \geq_{\mathbb{R}^{3}} 0_{\mathbb{R}^{3}}\right\}$, the admissible sets for (1.2) and (1.3), respectively.

Note that, $\operatorname{argmin}_{x \in S} f(x)$, the set of solutions for (1.2) can be written as

$$
\left\{x^{B}+\alpha \cdot\left(x^{1}-x^{B}\right) \mid \alpha \geq 0\right\},
$$

while for (1.3), one has

$$
\operatorname{argmin}_{x \in S^{\prime}} f_{2}(x)=\left\{x^{0}+\alpha \cdot\left(x^{2}-x^{0}\right) \mid \alpha \geq 0\right\},
$$

where $\left(x^{0}\right)^{T}=(16,13,4)$. 
Every vector $x \in \mathbb{R}^{n}$ is seen as a column vector and its transpose, denoted by $x^{T}=\left(x_{1}, \ldots, x_{n}\right) \in \mathbb{R}^{n}$, as a row vector. In particular, denote by $0_{\mathbb{R}^{n}}^{T}=(0, \ldots, 0) \in \mathbb{R}^{n}$ and by $e_{j}^{T}=(0, \ldots, 0,1,0, \ldots, 0)$ with 1 for the $j^{t h}$ position. The scalar product of $c \in \mathbb{R}^{n}$ and $x$ is given by

$$
c^{T} x=\sum_{i=1}^{n} c_{i} \cdot x_{i}
$$

For a matrix $A \in M_{m, n}(\mathbb{R})$ we denote by $A^{j} \in \mathbb{R}^{m}$ the $j$ th column of $A=\left(A^{j}\right)_{1 \leq j \leq n}$.

Let us remind two well-known relations in $\mathbb{R}^{n}$. Along with the already used one $x \geq_{\mathbb{R}^{n}} 0_{\mathbb{R}^{n}}$, that is $x_{i} \geq 0$, for all $i \in\{1, \ldots, n\}$, one has $x>_{\mathbb{R}^{n}} 0_{\mathbb{R}^{n}}$ iff $x_{i} \geq 0, \forall i$ and $\exists i_{0}$ with $x_{i_{0}}>0$. Also, $x \leq_{\mathbb{R}^{n}} y$ iff $y-x \geq 0_{\mathbb{R}^{n}}$, and $x>\mathbb{R}^{n} y$ iff $x-y>\mathbb{R}^{n} 0_{\mathbb{R}^{n}}$.

In the next section we give an easy example, a common type of situation when the solution is on the size of a polyhedron, i.e. the solution is not a unique point, in order to indicate when a subcase of step 4 of the new proposed algorithm occurs. In section 3, in step 4 of the Simplex Algorithm 2.0 are inserted the cases containing the bounded set of solutions as a convex combination and the unbounded set of solutions, respectively. These are summary formulated in Proposition 3.2. In its proof we indicate the direction, a vector denoted by $\bar{c}$, for the case of unboundedness (see also Lemma 3.1). The last section consists on the inductive construction of the unbounded set of solutions. Besides this, some necessary conditions on data are given in Proposition 4.5 so that the set of solutions to be boundedless.

Some basic motivations for this level of exposition could be finding the closest solution of (1.1) to a given point or, to find the point with integer coordinates, the closest one to the set of solutions.

\section{An Elementary Example for Multiple Solution}

Let us consider a simple linear programming problem with multiple solutions

$$
\left\{\begin{aligned}
-x_{1}-x_{2} & \longrightarrow \min \\
x_{1}+x_{2} & \leq 2 \\
x_{1} & \leq 1 \\
x_{1}, x_{2} \geq 0 &
\end{aligned}\right.
$$

From its standard form

$$
\left\{\begin{array}{c}
-x_{1}-x_{2} \longrightarrow \min \\
x_{1}+x_{2}+x_{3}=2 \\
x_{1}+x_{4}=1 \\
x_{1}, x_{2}, x_{3}, x_{4} \geq 0,
\end{array}\right.
$$

we have data $c^{T}=(-1,-1,0,0)$,

$$
A=\left(\begin{array}{cccc}
1 & 1 & 1 & 0 \\
1 & 0 & 0 & 1
\end{array}\right)=\left(A^{j}\right)_{1 \leq j \leq 4}, A^{j} \in \mathbb{R}^{2}, \text { and } b=\left(\begin{array}{l}
2 \\
1
\end{array}\right) .
$$

From the very first step, $\left\{A^{1}, A^{2}\right\}$ is an optimal basis. Using the usual simplex algorithm we get the solution $\left(x^{10}\right)^{T}=(1,1,0,0)$. Replacing $A^{4}$ with $A^{1}$ (see Figure 2 ), 
we obtain another solution $\left(x^{20}\right)^{T}=(0,2,0,1)$. Therefore, the solution for $(2.1)$ is the convex combination of $(1,1)^{T}$ and $(0,2)^{T}$, that is

$$
\left\{(1-\gamma, 1+\gamma)^{T} \mid \gamma \in[0,1]\right\} .
$$

\begin{tabular}{|c|c|c|c|}
\hline 1 & $A^{1}$ & $A^{2}$ & \\
\hline$A^{3}$ & 0 & 1 & -1 \\
\hline$A^{4}$ & 1 & -1 & 0 \\
\hline & 1 & 1 & \\
\hline
\end{tabular}

\begin{tabular}{|c|c|c|c|}
\hline 2 & $A^{4}$ & $A^{2}$ & \\
\hline$A^{3}$ & 0 & 1 & -1 \\
\hline$A^{1}$ & 1 & 1 & 0 \\
\hline & 1 & 2 & \\
\hline
\end{tabular}

Figure 2. Interchanging vectors

Our comment is that in contrast to this example, in Example 1.1 we cannot introduce $A^{5}$ in the optimal basis. It is a known fact that the optimum, if there is any, is attained at least in a point and at most on a hyperplane. How this should be done, eventually using tables?

Remark 2.1. It can be observed in the previous example(s) that $\max _{i \in \overline{\mathcal{B}}} \alpha_{i 0}=0$. This does not imply that the solution is not unique. For the following problem

$$
\left\{\begin{array}{rrr}
-x_{1}-x_{2}-2 x_{3} \longrightarrow \min & \\
x_{1}+x_{2}+x_{4} & =1 \\
x_{1}+x_{3}+x_{5} & =1 \\
x_{3} & +x_{6}=1 \\
x_{1}, \ldots, x_{6} \geq 0, &
\end{array}\right.
$$

we can obtain optimal bases $\left\{A^{2}, A^{3}, A^{5}\right\}, \alpha_{10}=0$ and $\left\{A^{1}, A^{2}, A^{3}\right\}, \alpha_{50}=0$, respectively with the unique solution $(0,1,1,0,0,0)$. In this case $\min _{j \in \mathcal{B}} \alpha_{0 j}=0$.

\section{The Simplex Algorithm 2.0}

Let

$$
S=\left\{x \in \mathbb{R}^{n} \mid A \cdot x=b, x \geq_{\mathbb{R}^{n}} 0_{n}\right\}
$$

be the admissible set related to (1.1).

In Step 4 we add the situations that provide the multiplicity solution.

Algorithm 2.0

Step 1. Chose primal admissible basis $B$ from $\left(A^{j}\right)_{1 \leq j \leq n}=A$;

Determine $\alpha_{0 j}\left(j \in \mathcal{B}:=\left\{j \in\{1,2, \ldots, n\} \mid A^{j} \in B\right\}\right)$ such that $b=\sum_{j \in \mathcal{B}} \alpha_{0 j} A^{j}$;

Put $s:=0$;

Step 2. Determine $\alpha_{i j}(i \in \overline{\mathcal{B}}:=\{1,2, \ldots, n\} \backslash \mathcal{B}, j \in \mathcal{B})$ such that $A^{i}=\sum_{j \in \mathcal{B}} \alpha_{i j} A^{j}$; 
Step 3. Compute

$$
\alpha_{i 0}=\sum_{j \in \mathcal{B}} \alpha_{i j} c_{j}-c_{i}, \forall i \in \overline{\mathcal{B}} ; \quad \alpha_{00}=\sum_{j \in \mathcal{B}} \alpha_{0 j} c_{j}
$$

Step 4. Look for the signs of the numbers $\alpha_{i 0}(i \in \overline{\mathcal{B}})$

a) $\forall i \in \overline{\mathcal{B}}, \alpha_{i 0} \leq 0$ then declare: "point $x^{s} \in \mathbb{R}^{n}$

$$
x_{j}^{s}= \begin{cases}\alpha_{0 j}, & j \in \mathcal{B} \\ 0, & j \in \overline{\mathcal{B}}\end{cases}
$$

is a solution for problem (1.1)" and $\alpha_{00}$ is the minimum value of the objective function;

a1) $\max _{i \in \overline{\mathcal{B}}} \alpha_{i 0}<0$, then declare:

$" x^{s}$ is the unique solution generated by $B "$.

a2) $\max _{i \in \overline{\mathcal{B}}} \alpha_{i 0}=0$ and $\min _{j \in \mathcal{B}} \alpha_{0 j}=0$, then declare:

" $x^{s}$ is the unique solution generated by $B$ ".

a3) $\max _{i \in \overline{\mathcal{B}}} \alpha_{i 0}=0$ and $\min _{j \in \mathcal{B}} \alpha_{0 j}>0$, then declare:

" $x^{s}$ is NOT the unique solution";

form the set

$$
\overline{\mathcal{B}}_{0}=\left\{i \in \overline{\mathcal{B}} \mid \alpha_{i 0}=0\right\}=\left\{\bar{i}_{1}, \bar{i}_{2}, \ldots, \bar{i}_{p}\right\} ;
$$

a3 $\alpha) \forall i \in \overline{\mathcal{B}}_{0}, \exists j \in \mathcal{B}$ such that $\alpha_{i j}>0$, chose an index $h \in \overline{\mathcal{B}}_{0}$;

$s:=s+1$;

If $s \leq p$ declare: "each element of $\operatorname{co}\left\{x^{0}, x^{s}\right\}$ is solution for problem (1.1)", and go to Step 6;

a3 $\beta) \exists \bar{i} \in \overline{\mathcal{B}}_{0}$ such that $\forall j \in \mathcal{B}, \alpha_{\bar{i} j} \leq 0$ then declare:

"the set of solutions is unbounded";

b) $\exists i \in \overline{\mathcal{B}}, \alpha_{i 0}>0$ then form the set

$$
\overline{\mathcal{B}}_{+}=\left\{i \in \overline{\mathcal{B}} \mid \alpha_{i 0}>0\right\}
$$

Step 5. $\forall i \in \overline{\mathcal{B}}_{+}$, Look for the signs of the numbers $\alpha_{i j}(j \in \mathcal{B})$

a) $\exists i \in \overline{\mathcal{B}}_{+}$such that $\forall j \in \mathcal{B}, \alpha_{i j} \leq 0$ then declare: "the objective function for (1.1) is unbounded from below over $S$, therefore (1.1) has no solution".

b) $\forall i \in \overline{\mathcal{B}}_{+}, \exists j \in \mathcal{B}$ such that $\alpha_{i j}>0$, chose an index $h \in \overline{\mathcal{B}}_{+}$;

Step 6. Choose an index $k \in \mathcal{B}$ such that $\alpha_{h k}>0$ and

$$
\frac{\alpha_{0 k}}{\alpha_{h k}}=\min \left\{\frac{\alpha_{0 j}}{\alpha_{h j}} \mid j \in \mathcal{B}, \alpha_{h j}>0\right\} ;
$$

Step 7. Replace in $B$, vector $A^{k}$ with $A^{h}$ then go to Step 2.

We are going to use the following lemma.

Lemma 3.1. Let $n>1$ and $c \in \mathbb{R}^{n}, c \neq 0_{\mathbb{R}^{n}}$. Suppose that

$$
\max _{i \in \overline{\mathcal{B}}} \alpha_{i 0}=0
$$

and let $\overline{\mathcal{B}}_{0}=\left\{i \in \overline{\mathcal{B}} \mid \alpha_{i 0}=0\right\}$. 
Suppose that exists $\bar{i} \in \overline{\mathcal{B}}_{0}$ such that $\alpha_{\bar{i} k} \leq 0, \forall k \in \mathcal{B}$.

Then, there exists $\bar{c} \in \mathbb{R}^{n}$ with $\bar{c}>_{\mathbb{R}^{n}} 0_{\mathbb{R}^{n}}$ such that

$$
\left\{\begin{array}{l}
c^{T} \bar{c}=0 \\
A \cdot \bar{c}=0_{\mathbb{R}^{m}}
\end{array}\right.
$$

Proof. We have

$$
\alpha_{\overline{i 0}}=\sum_{j \in \mathcal{B}} \alpha_{\overline{i j}} \cdot c_{j}-c_{\bar{i}}=0 .
$$

Define the following set of indexes $\mathcal{B}_{\bar{i} 0}=\left\{k \in \mathcal{B} \mid \alpha_{\bar{i} k}=0\right\}$ and the vector

$$
\bar{c}^{T}=\left(\bar{c}_{1}, \ldots, \bar{c}_{n}\right)
$$

by

$$
\bar{c}_{j}= \begin{cases}-\alpha_{\bar{i} j}, & j \in \mathcal{B} \backslash \mathcal{B}_{\bar{i} 0} \\ 1, & j=\bar{i} \\ 0, & \text { otherwise. }\end{cases}
$$

Clearly $\bar{c}>_{\mathbb{R}^{n}} 0_{\mathbb{R}^{n}}$ and $c^{T} \bar{c}=0$.

For $i \in \overline{\mathcal{B}}$ we have $A^{i}=\sum_{j \in \mathcal{B}} \alpha_{i j} A^{j}$ so, on the components

$$
a_{k i}=\sum_{j \in \mathcal{B}} \alpha_{i j} a_{k j}, k \in\{1, \ldots, m\},
$$

thus for $i=\bar{i}$

$$
a_{k \bar{i}}=\sum_{j \in \mathcal{B}} \alpha_{i j}^{\bar{B}} \cdot a_{k j}
$$

For $i \in\{1, \ldots, m\}$, by $(3.2)$ one has

$$
\sum_{j=1}^{n} a_{i j} \cdot \bar{c}_{j}=\sum_{j \in \mathcal{B} \cup\{\bar{i}\}} a_{i j} \cdot \bar{c}_{j}=\sum_{j \in \mathcal{B}} a_{i j} \cdot\left(-\alpha_{\bar{i} j}\right)+a_{i \bar{i}} \cdot 1=0 .
$$

Note that if $x^{1}, x^{2}$ are two different solutions of (1.1) then $x^{1}-x^{2}$ satisfies (3.1). If more, $x^{1}>_{\mathbb{R}^{n}} x^{2}$ then $\bar{c}$ can be taken $x^{1}-x^{2}$ and called direction of recession. We can define $\bar{c}$ except $\mathcal{B}_{\bar{i} 0}$, in a similar way

$$
\bar{c}_{j}=\left\{\begin{array}{ll}
-\alpha_{\bar{i} j}, & j \in \mathcal{B} \\
1, & j=\bar{i} \\
0, & \text { otherwise, }
\end{array} \quad \text { if } \overline{\mathcal{B}} \backslash\{\bar{i}\} \neq \emptyset,\right.
$$

else

$$
\bar{c}_{j}= \begin{cases}-\alpha_{\overline{i j}}, & j \in \mathcal{B} \\ 1, & j=\bar{i}\end{cases}
$$

Involving $\mathcal{B}_{\bar{i} 0}$ we underline on the components where $x^{1}>_{\mathbb{R}^{n}} x^{2}$.

As regards the three situations of Step 4 of the algorithm, when there is a unique solution, a bounded set of solutions (but not a singleton) or the unbounded set of solutions, we formulate the following result. 
Proposition 3.2. Suppose that $x^{0}=x^{B}$ is a solution for (1.1) obtained in Step 4 a) of the algorithm and that $B$ is the optimal basis. The following implications apply:

1. If $\max _{i \in \overline{\mathcal{B}}} \alpha_{i 0}<0$, then there is no other solution generated by the optimal basis $B$.

2. If $\max _{i \in \overline{\mathcal{B}}} \alpha_{i 0}=0$ and $\min _{j \in \mathcal{B}} \alpha_{0 j}=0$, then there is no other solution generated by the optimal basis $B$.

3. If $\max _{i \in \overline{\mathcal{B}}} \alpha_{i 0}=0$ and $\min _{j \in \mathcal{B}} \alpha_{0 j}>0$, then

$$
\left\{x^{0}\right\} \subsetneq \operatorname{argmin}_{x \in S} f(x)
$$

if more

a3 $\alpha$ ) for some $\bar{i} \in \overline{\mathcal{B}}_{0}=\left\{i \in \overline{\mathcal{B}} \mid \alpha_{i 0}=0\right\}$, there exists $k \in \mathcal{B}$ such that $\alpha_{\bar{i} k}>0$, then the set of solutions contains

$$
\operatorname{co}\left\{x^{0}, x^{1}, \ldots, x^{p}\right\},
$$

with $p \leq \operatorname{card} \overline{\mathcal{B}}_{0}$, where

$$
\overline{\mathcal{B}}_{0+}=\left\{i \in \overline{\mathcal{B}}_{0} \mid \exists k \in \mathcal{B} \text { such that } \alpha_{i k}>0\right\}
$$

a3 $\beta$ ) for some $\bar{i} \in \overline{\mathcal{B}}_{0}, \alpha_{\bar{i} k} \leq 0, \forall k \in \mathcal{B}$, then the set of solutions is (convex) unbounded.

Proof. Let $f(x)=c^{T} x$.

1. The proof is adapted from the literature (see $[3,4])$, inspired by the variation of the objective function when a vector is replaced from the primal basis. Suppose $x^{B^{\prime}} \in \operatorname{argmin}_{x \in S} f(x), x^{B^{\prime}} \neq x^{0}$.

Let $h \in \overline{\mathcal{B}}$. Passing from the optimal basis $B$ to the other optimal one $B^{\prime}$, suppose that $B^{\prime}$ is constructed by replacing a vector $A^{k}$ with $A^{h}$. Let $\mathcal{B}^{\prime}=(\mathcal{B} \backslash\{k\}) \cup\{h\}$.

Let $\theta=\frac{\alpha_{0 k}}{\alpha_{h k}}$. The new coordinates are

$$
\alpha_{0 j}^{\prime}= \begin{cases}\alpha_{0 j}-\theta \cdot \alpha_{h j}, & j \neq k \\ \theta, & j=h\end{cases}
$$

Denote by $\left(x^{B^{\prime}}\right)^{T}=\left(x_{1}^{B^{\prime}}, \ldots, x_{n}^{B^{\prime}}\right) \in \mathbb{R}^{n}$, where $x_{j}^{B^{\prime}}=\left\{\begin{array}{ll}\alpha_{0 j}^{\prime}, & j \in \mathcal{B}^{\prime} \\ 0, & j \in \overline{\mathcal{B}}^{\prime}\end{array}\right.$.

For $x^{B^{\prime}}$ the value of the objective function is

$$
\begin{aligned}
f\left(x^{B^{\prime}}\right) & =\alpha_{00}=c^{T} x^{B^{\prime}}=\sum_{j \in \mathcal{B}^{\prime}} \alpha_{0 j}^{\prime} \cdot c_{j}=\sum_{j \in \mathcal{B}}\left[\alpha_{0 j}-\theta \cdot \alpha_{h j}\right] \cdot c_{j}+\theta \cdot c_{h} \\
& =\sum_{j \in \mathcal{B}} \alpha_{0 j} \cdot c_{j}+\theta \cdot\left[c_{h}-\sum_{j \in \mathcal{B}} \alpha_{h j} \cdot c_{j}\right]=\alpha_{00}+\theta \cdot\left(c_{h}-z_{h}\right),
\end{aligned}
$$

where $z_{h}=\sum_{j \in \mathcal{B}} \alpha_{h j} \cdot c_{j}$. We obtained

$$
\alpha_{00}=\alpha_{00}+\theta \cdot\left(c_{h}-z_{h}\right)
$$

Now we have

$$
0<-\alpha_{h 0}=c_{h}-z_{h}=c_{h}-\sum_{j \in \mathcal{B}} \alpha_{h j} \cdot c_{j}=0
$$


thus we get the contradiction.

2. Let $\bar{i} \in \overline{\mathcal{B}}_{0+}$. By replacing vector $A^{\bar{i}}$ with $A^{\bar{j}}, \alpha_{0 \bar{j}}=0$, the value of the objective function does not change

$$
f\left(x^{0}\right)-\alpha_{\bar{i} 0} \cdot \frac{\alpha_{0 \bar{j}}}{\alpha_{\bar{i} \bar{j}}}=f\left(x^{0}\right),
$$

and $\mathcal{B}^{\prime}=(\mathcal{B} \backslash\{\bar{j}\}) \cup\{\bar{i}\}, \alpha_{0 \bar{i}}=0$.

3.

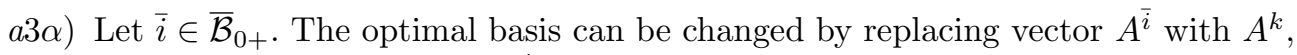
to obtain another solution $x^{1}$. We have

$$
f\left(x^{1}\right)=f\left(x^{0}\right)-\alpha_{\bar{i} 0} \cdot \frac{\alpha_{0 k}}{\alpha_{\bar{i} k}}=f\left(x^{0}\right)=\alpha_{00},
$$

and that $(1-\gamma) \cdot x^{0}+\gamma \cdot x^{1},(\gamma \in[0,1])$ is solution for (1.1) as well. It is easy to check that $(1-\gamma) \cdot x^{0}+\gamma \cdot x^{1} \in S$ and by the linearity of $f$ we obtain $c^{T}\left[(1-\gamma) \cdot x^{0}+\gamma \cdot x^{1}\right]=(1-\gamma) \cdot c^{T} x^{0}+\gamma \cdot c^{T} x^{1}=c^{T} x^{0}$.

a3 $\beta$ ) Since $x^{0}$ is a solution given by the (classic) simplex algorithm, it is an extremal point. Let $\bar{i} \in \overline{\mathcal{B}}_{0}$ be fixed such that $\alpha_{\bar{i} k} \leq 0, \forall k \in \mathcal{B}$. From Lemma 3.1 there exists $\bar{c}>_{\mathbb{R}^{n}} 0_{\mathbb{R}^{n}}$ with $c^{T} \bar{c}=0$ and $A \cdot \bar{c}=0_{\mathbb{R}^{m}}$. Let

$$
\bar{x}=x^{0}+\bar{c}, \text { with } \bar{c}=\sum_{j \in \mathcal{B} \cup\{\bar{i}\} \backslash \mathcal{B}_{\bar{i} 0}} \bar{c}_{j} e_{j},
$$

where $\mathcal{B}_{\bar{i} 0}=\left\{k \in \mathcal{B} \mid \alpha_{\bar{i} k}=0\right\}$ is defined in the proof of Lemma 3.1.

We claim that this direction $\bar{x}-x^{0}$, gives the unboundedness of the solution set. Firstly, we prove that $x^{0}+\alpha \cdot\left(\bar{x}-x^{0}\right)$ is admissible. We have

$$
A \cdot x^{0}+\alpha \cdot A \cdot\left(\bar{x}-x^{0}\right)=b+\alpha \cdot A \cdot \bar{c}=b .
$$

Since $\bar{x}>_{\mathbb{R}^{n}} x^{0}$, it follows that for $\alpha \geq 0$ we have $x^{0}+\alpha \cdot\left(\bar{x}-x^{0}\right) \geq_{\mathbb{R}^{n}} 0_{\mathbb{R}^{n}}$. Now,

$$
f\left(x^{0}+\alpha \cdot\left(\bar{x}-x^{0}\right)\right)=c^{T} x^{0}+\alpha \cdot c^{T}\left(\bar{x}-x^{0}\right)=c^{T} x^{0} .
$$

The statement about the convex hull containment, generated by step $a 3 \alpha$ is a partial result. At this point we were not able to generate the complete solution.

Remark 3.3. The following are related to the case $a 3 \alpha$ from Step 4 of the Simplex Algorithm 2.0. If for some index $\bar{i} \in \overline{\mathcal{B}}_{0}=\left\{i \in \overline{\mathcal{B}} \mid \alpha_{i 0}=0\right\}$, there exists another index $k \in \mathcal{B}$ such that $\alpha_{\bar{i} k}>0$, then we cannot conclude on the boundedness of the solution set of (1.1). Precisely, two different situations can occur, the set of solutions could be bounded or boundedless, considering the following two problems:

1.

$$
\left\{\begin{array}{c}
x_{1}+x_{2} \quad+x_{4} \longrightarrow \min \\
x_{1}+x_{3}-x_{4}=2 \\
x_{2}-x_{3}+2 x_{4}=3 \\
x_{1}, x_{2}, x_{3}, x_{4} \geq 0
\end{array}\right.
$$


with the complete solution (convex) bounded. From data $c^{T}=(1,1,0,1)$, $A=\left(\begin{array}{cccc}1 & 0 & 1 & -1 \\ 0 & 1 & -1 & 2\end{array}\right)=\left(A^{j}\right)_{1 \leq j \leq 4}, \quad$ and $\quad b=\left(\begin{array}{l}2 \\ 3\end{array}\right), B=\left\{A^{1}, A^{2}\right\}$ is an optimal basis, $\left(x^{0}\right)^{T}=(2,3,0,0)$ is a solution generated by $B$, and $\overline{\mathcal{B}}_{0}=\{3,4\}$. After replacing $A^{1}$ with $A^{3}$ we obtain solution $\left(x^{1}\right)^{T}=(0,5,2,0)$, replacing $A^{2}$ with $A^{4}$ we get solution $\left(x^{2}\right)^{T}=(0,0,7,5)$, replacing $A^{3}$ with $A^{1}$ we obtain solution $\left(x^{3}\right)^{T}=(7 / 2,0,0,3 / 2)$.

Problem (3.3) reduces to the system of inequalities

$$
\left\{\begin{array}{r}
x_{3}-x_{4} \leq 2 \\
-x_{3}+2 x_{4} \leq 3 \\
x_{3}, x_{4} \geq 0
\end{array}\right.
$$

that represents $c o\{(0,0),(2,0),(7,5),(0,3 / 2)\}$.

The set of solutions for problem (3.3) is

$$
\operatorname{co}\left\{x^{0}, x^{1}, x^{2}, x^{3}\right\} \text {. }
$$

2.

$$
\left\{\begin{array}{c}
x_{1}+x_{2} \quad \longrightarrow \min \\
x_{1}+x_{3}-x_{4}=2 \\
x_{2}-x_{3}+x_{4}=3 \\
x_{1}, x_{2}, x_{3}, x_{4} \geq 0
\end{array}\right.
$$

with the complete solution (convex) boundedless. From data $c^{T}=(1,1,0,0)$, $A=\left(\begin{array}{cccc}1 & 0 & 1 & -1 \\ 0 & 1 & -1 & 1\end{array}\right)=\left(A^{j}\right)_{1 \leq j \leq 4}, \quad$ and $\quad b=\left(\begin{array}{l}2 \\ 3\end{array}\right), B=\left\{A^{1}, A^{2}\right\}$

is an optimal basis with solution $\left(x^{0}\right)^{T}=(2,3,0,0)$ and $\overline{\mathcal{B}}_{0}=\{3,4\}$. After replacing $A^{1}$ with $A^{3}$ we obtain solution $\left(x^{1}\right)^{T}=(0,5,2,0)$, and the case $a 3 \beta$ with $\bar{i}=4$ (see Figure 3 ). The set of solutions is unbounded.

\begin{tabular}{|c|c|c|c|}
\hline 1 & $A^{1}$ & $A^{2}$ & \\
\hline$A^{3}$ & 1 & -1 & 0 \\
\hline$A^{4}$ & -1 & 1 & 0 \\
\hline & 2 & 3 & \\
\hline
\end{tabular}

\begin{tabular}{|c|c|c|c|}
\hline 2 & $A^{3}$ & $A^{2}$ & \\
\hline$A^{1}$ & 1 & 1 & 0 \\
\hline$A^{4}$ & -1 & 0 & 0 \\
\hline & 2 & 5 & \\
\hline
\end{tabular}

Figure 3. $a 3 \beta$ 
Since $x^{0}$ and $x^{1}$ are not comparable in $\mathbb{R}^{4}$, the direction of recession cannot be taken $x^{0}-x^{1}$. The set of solutions contains $\operatorname{co}\left\{x^{0}, x^{1}\right\}$. By the proof of Lemma 3.1 , with $\mathcal{B}=\{2,3\}$ and $\mathcal{B}_{40}=\{2\}$, we have

$$
\mathcal{B} \cup\{4\} \backslash \mathcal{B}_{40}=\{2,3\} \cup\{4\} \backslash\{2\}=\{3,4\},
$$

therefore we get $\bar{c}^{T}=(0,0,1,1)$. Problem (3.4) reduces to the system of inequalities

$$
\left\{\begin{array}{l}
x_{3}-x_{4} \leq 2 \\
-x_{3}+x_{4} \leq 3 \\
x_{3}, x_{4} \geq 0
\end{array}\right.
$$

The complete solution is

$$
\left\{x^{0}+\alpha \cdot \bar{c} \mid \alpha \geq 0\right\}=\left\{(2,3, \alpha, \alpha)^{T} \mid \alpha \geq 0\right\} .
$$

In Example 1.1 was underlined the case for a3 $\beta$. Going back to it, via Lemma 3.1, we have $\mathcal{B}=\{1,2,3\}, \overline{\mathcal{B}}_{0}=\{5\}, \bar{i}=5, \mathcal{B}_{50}=\{3\}$, therefore $\bar{c}^{T}=(1,1,0,0,1,0)$.

\section{Comments}

In Section 2, we had the situation in Step 4, a3 $\alpha$. In order to follow easily the description of indexes we give this obvious example.

Example 4.1. Consider the linear programming problem

$$
\left\{\begin{array}{c}
-x_{1}-x_{2}-x_{3} \longrightarrow \min \\
x_{1}+x_{2}+x_{3}=1 \\
x_{1}, x_{2}, x_{3} \geq 0
\end{array}\right.
$$

in its standard form. Data are $c^{T}=(-1,-1,-1), A=\left(\begin{array}{lll}1 & 1 & 1\end{array}\right)=\left(A^{j}\right)_{1 \leq j \leq 3}$, and $b=1$.

$A^{3}$ can be considered as basis, that is optimal. We are in the case a $3 \alpha, \overline{\mathcal{B}}=\{2,3\}=$ $\overline{\mathcal{B}}_{0+}$. Simplex algorithm provides the solution $x^{0}=(0,0,1)$. After replacing successively $A^{3}$ with $A^{1}$, we get $x^{1}=(1,0,0)$, then $A^{1}$ with $A^{2}$, we get $x^{2}=(0,1,0)$. Therefore the solution for (4.1) is $\operatorname{co}\left\{x^{0}, x^{1}, x^{2}\right\}$.

When the size of the problem is not large, finding all the extremal points is not difficult. What remains to be done when it is not possible to introduce $A^{\bar{i}}$ in the optimal basis? A possibility is to combine the classical solution with one provided by Matlab command linprog. So, when case a $3 \beta$ shows up, the complete solution contains the set

$$
\left\{x^{0}+\alpha \cdot\left(x^{1}-x^{0}\right) \mid \alpha \geq 0\right\},
$$

with $x^{1} \neq x^{0}$, a solution that can be obtained unconventionally with respect to the algorithm. More questions arise then.

Similar to Example 1.1, we consider the following trivial one. 
Example 4.2. Being given the linear programming problem

$$
\left\{\begin{array}{l}
x_{1}-x_{2} \longrightarrow \min \\
-x_{1}+x_{2} \leq 0 \\
x_{1}, x_{2} \geq 0
\end{array}\right.
$$

from its standard form

$$
\left\{\begin{array}{c}
x_{1}-x_{2} \longrightarrow \min \\
-x_{1}+x_{2}+x_{3}=0 \\
x_{1}, x_{2}, x_{3} \geq 0
\end{array}\right.
$$

the simplex algorithm provides the solution $(0,0,0)^{T}$, thus for $(4.2)$ we take $\left(x^{0}\right)^{T}=$ $(0,0)$. We are in the case a3 $\beta$. Another solution for $(4.2)$ is $\left(x^{1}\right)^{T}=(1,1)$, (or the one provided by Matlab $(84.7173,84.7173)^{T}$ can be used) therefore the set of solutions is

$$
\left\{(\alpha, \alpha)^{T} \mid \alpha \geq 0\right\} .
$$

Worth noted or not, its dual problem has a unique solution -1 .

What is the algebraic dimension of the unboundedness in the case a3 $\beta$ ? At least empirically, with no intention to prove that this is true at this stage, we claim that it would be $\operatorname{card} \overline{\mathcal{B}}_{0}^{\beta}$ where $\overline{\mathcal{B}}_{0}^{\beta}=\overline{\mathcal{B}}_{0-} \cup\left\{\bar{i} \in \overline{\mathcal{B}}_{0+} \mid \overline{\mathcal{B}}_{0-} \neq \emptyset\right\}$, with $\overline{\mathcal{B}}_{0-}=\left\{i \in \overline{\mathcal{B}}_{0} \mid \alpha_{i k} \leq 0, \forall k \in \mathcal{B}\right\}$. Clearly, if $\overline{\mathcal{B}}_{0-}=\emptyset$ then $\overline{\mathcal{B}}_{0}^{\beta}=\emptyset$, and the set of solutions is bounded, that is conform to the algorithm. Also, $\left[\overline{\mathcal{B}}_{0-}=\{\bar{i}\}\right.$ and $\left.\overline{\mathcal{B}}_{0+}=\emptyset\right]$ iff $\operatorname{card} \overline{\mathcal{B}}_{0}^{\beta}=1$. Example 1.1 involves $\overline{\mathcal{B}}_{0}=\{5\}=\overline{\mathcal{B}}_{0-}, \overline{\mathcal{B}}_{0+}=\emptyset, \overline{\mathcal{B}}_{0}^{\beta}=\{5\}$.

Example 4.3. Being given the linear programming problem

$$
\left\{\begin{array}{l}
x_{1}-x_{2}-x_{3} \longrightarrow \min \\
-x_{1}+x_{2}+x_{3} \leq 0 \\
x_{1}, x_{2}, x_{3} \geq 0
\end{array}\right.
$$

from its standard form, the simplex algorithm provides the solution $\left(x^{0}\right)^{T}=(0,0,0)$. We are in the case a $\beta$, with $\operatorname{card} \overline{\mathcal{B}}_{0}^{\beta}=2$, given by $\mathcal{B}=\{1\}, \overline{\mathcal{B}}=\{2,3\}=$ $\overline{\mathcal{B}}_{0}=\overline{\mathcal{B}}_{0-}, \overline{\mathcal{B}}_{0+}=\emptyset, \overline{\mathcal{B}}_{0}^{\beta}=\{2,3\}$. Other solutions for $(4.3)$ are $\left(x^{1}\right)^{T}=(1,0,1)$ and $\left(x^{2}\right)^{T}=(1,1,0)$, or possibly other two provided by Matlab using two different starting points. By this eventually, we mean the logistic part of exposure that interfere. For example, along with data $c=\left[\begin{array}{lll}1 & -1 & -1\end{array}\right], A=\left[\begin{array}{lll}-1 & 1 & 1\end{array}\right], b=0$, and $l b=z \operatorname{eros}(1,3)$, if (admissible) starting points $x 0=\left[\begin{array}{lll}1 & 1 & 1\end{array}\right]$ and $x 0=\left[\begin{array}{lll}2 & 2 & 2\end{array}\right]$ are chosen, then using command linprog as $x=\operatorname{linprog}(c, A, b,[],[], l b,[], x 0)$ solutions provided (with some care to the approximations) are $\left(x^{1}\right)^{T}=(0.6667,0.3333,0.3333)$ and $\left(x^{2}\right)^{T}=(1.3333,0.6667,0.6667)$, respectively. More accurate, rats $(x)$ returns $\left(x^{1}\right)^{T}=(2 / 3,1 / 3,1 / 3)$ and $\left(x^{2}\right)^{T}=(4 / 3,2 / 3,2 / 3)$, respectively. By this random choice $\operatorname{rank}\left(x^{0}, x^{1}, x^{2}\right)=1$. By using $x 0=[312]$ as starting point Matlab returns solution $\left(x^{3}\right)^{T}=(1.8681,0.3899,0.4783)$, getting $\operatorname{rank}\left(x^{0}, x^{1}, x^{3}\right)=2=\operatorname{card} \overline{\mathcal{B}}_{0}^{\beta}$. The set of solutions is

$$
\left\{\left(\alpha_{1}+\alpha_{2}, \alpha_{1}, \alpha_{2}\right)^{T} \mid \alpha_{1}, \alpha_{2} \geq 0\right\} .
$$


By the examples above we claim that in the case a $3 \beta$ we have

$$
\operatorname{argmin}_{x \in S} f(x) \supseteq\left\{\left(1-\sum_{k \in \overline{\mathcal{B}}_{0}^{\beta}} \alpha_{k}\right) \cdot x^{0}+\sum_{k \in \overline{\mathcal{B}}_{0}^{\beta}} \alpha_{k} \cdot x^{k} \mid \alpha_{k} \geq 0\right\},
$$

where $x^{k}, k \in \overline{\mathcal{B}}_{0}^{\beta}$, are different solutions, such that $\operatorname{rank}\left(x^{k}\right)_{k \in \overline{\mathcal{B}}_{0}^{\beta}}=\operatorname{card} \overline{\mathcal{B}}_{0}^{\beta}$, eventually obtained by Matlab using different starting points.

For $\operatorname{card} \overline{\mathcal{B}}_{0}^{\beta}=1$ the result is contained in the following lemma.

Lemma 4.4. ([3]) Let $M \subset \mathbb{R}^{n}$ be nonempty, convex, closed. Then

$M$ is unbounded $\Longleftrightarrow \exists \bar{x} \in \mathbb{R}^{n} \backslash\left\{0_{n}\right\}$ such that $M+\alpha \cdot \bar{x} \subseteq M, \forall \alpha \in \mathbb{R}_{+}$.

We can use the classic result above with $\bar{x}:=x^{1}-x^{0}$ and $\alpha:=\alpha_{1}$. Then, we can repeatedly apply Lemma 4.4 for $M=\operatorname{argmin}_{x \in S} f(x)$ :

$$
\operatorname{argmin}_{x \in S} f(x)+\alpha_{1} \cdot\left(x^{1}-x^{0}\right)+\alpha_{2} \cdot\left(x^{2}-x^{0}\right)+\cdots+\alpha_{k} \cdot\left(x^{k}-x^{0}\right) \subseteq \operatorname{argmin}_{x \in S} f(x) .
$$

To end up with our comments, for case a $3 \beta$, we have the following assertion.

Proposition 4.5. For the canonical form of the linear programming problem with data $c \in \mathbb{R}^{n}, A \in M_{m, n}(\mathbb{R})$, and $b \in \mathbb{R}^{m}$

$$
\left\{\begin{array}{l}
f(x)=c^{T} x \longrightarrow \min \\
A \cdot x \leq \mathbb{R}^{m} b \\
x \geq \mathbb{R}^{n} 0_{\mathbb{R}^{n}}
\end{array}\right.
$$

if the solution set is unbounded, then there exist $I \subseteq\{1, \ldots, m\}$ and $d \in \mathbb{R}_{-}^{I}$ such that

$$
f(x)=\sum_{i \in I} d_{i} \sum_{j=1}^{n} a_{i j} x_{j}
$$

consequently

$$
\left\{c,\left(a_{i j}\right)_{j}\right\}_{i \in I} \text { is a linearly dependent algebraic system. }
$$

Proof. The admissible set $S^{\prime}=\left\{x \in \mathbb{R}^{n} \mid A \cdot x \leq_{\mathbb{R}^{m}} b, x \geq_{\mathbb{R}^{n}} 0_{\mathbb{R}^{n}}\right\}$ must be unbounded. By Lemma 4.4, there exists $\bar{x} \in \mathbb{R}^{n} \backslash\left\{0_{\mathbb{R}^{n}}\right\}$ such that

$$
\operatorname{argmin}_{x \in S^{\prime}} f(x)+\alpha \cdot \bar{x} \subseteq \operatorname{argmin}_{x \in S^{\prime}} f(x), \forall \alpha \in \mathbb{R}_{+} .
$$

Let $x^{0}$ be an arbitrary solution for (4.4). By Karush-Kuhn-Tucker theorem, there exist an active set of indexes $I$ and $v \in \mathbb{R}_{+}^{I}$ such that

$$
\left[\nabla f\left(x^{0}\right)+\sum_{i \in I} v_{i} \cdot \nabla g_{i}\left(x^{0}\right)\right]^{T}\left(x-x^{0}\right) \geq 0, \forall x \in S^{\prime},
$$

where $g_{i}(x)=(A \cdot x-b)_{i}, i \in I$.

Let $x^{\prime}$ be another solution. Denote by

$$
d_{\alpha, x^{0}}=\left\{x^{0}+\alpha \cdot\left(x^{\prime}-x^{0}\right) \mid \alpha \geq 0\right\} .
$$


We have $d_{\alpha, x^{0}} \cap \operatorname{int}_{a} S^{\prime} \neq \emptyset$, (here $\operatorname{int}_{a} Y$ denotes the algebraic interior of a set $Y$ ). Let $\bar{x}^{0} \in d_{\alpha, x^{0}} \cap \operatorname{int}_{a} S^{\prime}$. Then

$$
\nabla f\left(\bar{x}^{0}\right)+\sum_{i \in I} v_{i} \cdot \nabla g_{i}\left(\bar{x}^{0}\right)=0_{\mathbb{R}^{n}}^{T}
$$

that is

$$
c^{T}=-\sum_{i \in I} v_{i} \cdot \nabla g_{i}\left(\bar{x}^{0}\right) .
$$

Integrating on the segment $\left[\bar{x}^{0}, x^{\prime}\right]$, for $v=\left(v_{i}\right)_{i \in I}$ we obtain

$$
f+v^{T}\left[(A \cdot x)_{i}\right]_{i \in I}=\text { constant }
$$

By the linearity of both terms, the constant is 0 . Now take $d:=-v$.

One more time back to Example 1.1, there exist $I=\{1,3\}$ and $d=(-4,-1) \in \mathbb{R}_{-}^{I}$ such that $f\left(x_{1}, x_{2}, x_{3}\right)=3 x_{1}-3 x_{2}-4 x_{3}=(-4) \cdot\left(-x_{1}+x_{2}+x_{3}\right)+(-1) \cdot\left(x_{1}-x_{2}\right)$, consequently $\left(c^{\prime}\right)^{T}=(3,-3,-4), a_{1}^{T}=(-1,1,1)$, and $a_{3}^{T}=(1,-1,0)$ are linearly dependent.

The reverse implication is not true (see section 2), unless $S^{\prime}$ is unbounded. This is still not sufficient as one can see from this one with no solution

$$
\left\{\begin{array}{l}
x_{1}-x_{2} \longrightarrow \min \\
x_{1}-x_{2} \leq 0 \\
x_{1}, x_{2} \geq 0
\end{array}\right.
$$

The nonemptiness of the solution set must be imposed. Not even so would be the enough, since the following problem

$$
\left\{\begin{array}{c}
x_{1}-x_{2} \longrightarrow \min \\
-x_{1}+x_{2} \leq 0 \\
x_{2} \leq 1 \\
x_{1}, x_{2} \geq 0
\end{array}\right.
$$

has a bounded solution set. Maybe, if the direction of the unboundedness for $S^{\prime}$, say $\tilde{c}$, to overlap on the vector $c$, in some sense, for example

$$
c^{T} \tilde{c}=0
$$

and $A \cdot \tilde{c}=0_{\mathbb{R}^{m}}$, could be the challenge.

Acknowledgements. I would like to express my appreciation to Professor Wolfgang W. Breckner for his lectures on Operations Research. I thank Brigitte Breckner for her remarks on a previous version of the manuscript. Nevertheless, I am grateful to the reviewer for pointing out errors and suggesting the list of references on interior-point method. 


\section{References}

[1] Breckner, E.B., De la poliedre la jocuri matriceale, Editura Fundaţiei pentru Studii Europene, Cluj-Napoca, 2007.

[2] Breckner, E.B., Popovici, N., Probleme de cercetare operaţională, Editura Fundaţiei pentru Studii Europene, Cluj-Napoca, 2006.

[3] Breckner, W.W., Cercetări operaţionale, curs litografiat, Cluj-Napoca, 1974.

[4] Dantzig, G.B., Linear Programming and Extensions, Princeton University Press, 1963.

[5] Dantzig, G.B., Linear Programming, Oper. Res., 50(2002), no. 1, 42-47.

[6] Darvay, Zs., New Interior Point Algorithms in Linear Programming, Advanced Modeling and Optimization, 5(2003), no. 1, 51-92.

[7] Griffin, C., Linear Programming: Penn State Math 484 Lecture Notes, 2009-2014, http://www.personal.psu.edu/cxg286/Math484_V1.pdf

[8] Robere, R., Interior Point Methods and Linear Programming, http://www.cs.toronto.edu/ robere/paper/interiorpoint.pdf

[9] Roos, C., Terlaky, T., Vial, J.-Ph., Theory and Algorithms for Linear Optimization, Springer, New York, USA, 2005.

[10] Wright, S.J., Primal-Dual Interior-Point Methods, SIAM, Philadelphia, USA, 1997.

[11] Ye, Y., Interior Point Algorithms, Theory and Analysis, John Wiley \& Sons, Chichester, UK, 1997.

[12] Excel, http://www.solver.com/excel-solver-linear-programming

[13] Matlab, http://www.mathworks.com/help/optim/ug/linprog.html

[14] Octave, http://www.obihiro.ac.jp/ suzukim/masuda/octave/html3/octave_125.html

[15] Octave, http://octave-online.net/

Marcel Bogdan

"Petru Maior" University, Faculty of Sciences and Letters

1 , N. Iorga Street,

540088 Târgu Mureş, Romania

e-mail: marcel.bogdan@science.upm.ro 